\title{
Ceftriaxone, ofloxacin or both in the treatment of enteric
}

\section{fever.}

\author{
Rishi Khatri ${ }^{l}$, Rajeeb Kumar Deo ${ }^{1}$, Binod Karki ${ }^{l}$, Arun Sharmal.
}

${ }^{1}$ Department of Medicine, Shree Birendra Hospital.

\begin{abstract}
Introduction: Enteric fever is a common diagnosis in febrile patients admitting to the hospital of Nepal. The treatment of enteric fever, though looks straightforward, in recent times has been complicated by emerging resistance to various antibiotics including fluoroquinolones and cephalosporins.
\end{abstract}

Methods: This was a randomized clinical study done at Shree Birendra Hospital from January 2011 to December 2011.Patients with with high grade fever , headache, relative bradycardia, abdominal pain, diarrhoea or constipation with normal to low leukocyte count were clinically diagnosed as Enteric fever and were randomized to either ofloxacin or ceftriaxone group, both well accepted first line treatment for enteric fever and observed for at least five days before the addition of the second drug that is ceftriaxone in ofloxacin group and ofloxacin in ceftriaxonegroup and the response to the drug was noted in terms of defervescence of fever.

Results: Total 50 patients were enrolled, 25 in each treatment group. After five days, six patients in Ceftriaxone group needed addition of Ofloxacin and two patients in Ofloxacin group needed Ceftriaxone. The average fever defervescence time in Ceftriaxone group was 3.88 days and in Ofloxacin group was 3.04 days. All patients were afebrile by 9 days of antibiotics.

Conclusions: Fever defervescence time with Ofloxacin was lesser than ceftriaxone group and need of supplementary antibiotic is lesser in ofloxacine group. So, ofloxacin can be recommended as 1st line drug for enteric fever.

Keywords: ceftriaxone; enteric fever; fever defervescence; ofloxacin

\section{INTRODUCTION}

Enteric fever is characterized by systemic illness with fever and abdominal pain ${ }^{1}$.The incidence of enteric fever has declined greatly with the provision of clean water and good sewage systems in Europe and the USA since the early $20^{\text {th }}$ century ${ }^{2}$ but the disease remains a serious publichealth problem in developing countries ${ }^{3}$.Enteric fever is common diagnosis in day to day practice in Kathmandu which can be attributed to poor personal hygiene, poor sanitation, and poor quality of water supply. The treatment of enteric fever at the outset may look straightforward with various classes of effective antibiotics available for use but the emerging resistance is a big problem.

The problem may be choice of too many and choosing appropriate antibiotic can be a challenge at times. Treatment of enteric fever has been complicated by the development and rapid dissemination of typhoidal organisms resistant to ampicillin, trimethoprim-sulfamethoxazole, and chloramphenicol. In recent years, development of resistance to fluoroquinolones as well as some extent to cephalosporins has resulted in more challenges ${ }^{4-5}$.

\section{Correspondence:}

Col. Dr. Rishi Khatri

Department of Medicine, Shree Birendra Hospital, Kathmandu Nepal

Email: khatririshi@ymail.com 
In this setting, it is really challenging to decide which antibiotics to initiate and how long to wait before adding the second drug for the treatment. In our study, we have tried to find the efficacy of ofloxacin and ceftriaxone alone or in combination for the treatment of enteric fever.

\section{METHODS}

The randomized clinical study was conducted at Shree Birendra Hospital for a period of one year from January 2011 to July 2011, which comprised of fifty consecutive patients admitted with clinical diagnosis of Enteric fever as suggested by high grade fever, headache, relative bradycardia, abdominal pain, diarrhoea orconstipation with normal to low leukocyte count. Blood culture was sent from each patient. All patients above 15 years old with the abovementioned clinical features were included in the study and any patient already taking antibiotics from outside setting was excluded. The permission was taken from the hospital administration and consent obtained from the patients. They were randomly divided in two groups to receive either intravenous Ceftriaxone 2 g per day or intravenous ofloxacin 400 mgper day, which are both, approved first line therapy for enteric fever. Patients were observed for at least five days for the fever defervescence before adding on the second antibiotic (intravenousOfloxacin in patients receiving Ceftriaxone and intravenous Ceftriaxone in a patients receiving Ofloxacin). Once the patient became afebrile for about 48 hours they were switched to oral ofloxacin 800 $\mathrm{mg}$ per day or oral Cefixime $800 \mathrm{mg}$ per day from their respective iv preparations. All patients were planned for total of 14 days of therapy. The patients were discharged after 14 days and asked to follow up after one week in medical OPD. The data were tabulated and analysed using chi-sqaire and t test as indicated using SPSS 17.1 software.

\section{RESULTS}

There were 25 patients in Ceftriaxone group and 25 patients in Ofloxacin group. The baseline characteristics in both the treatment arm were comparable in the study (Table 1). The mean age of the patient in the ofloxacin group was 28.92 and that of the ceftriaxone grop was 31.36 which was not statistically significant $(\mathrm{p}=0.45)$.The distribution of Sex (male), duration of Fever (days), and symptoms like Headache, Abdominal pain, diarrhoea, Constipation, Cough and Blood culture positivity were not different in both the group indicating that both the groups were comparable (Figure 1). The fever duration at the time of presentation to hospital for patients in Ceftriaxone group and Ofloxacin group was 8.6 days and 6.04 days respectively. Six patients in Ceftriaxone group needed addition of Ofloxacin after five days and two patients in Ofloxacin group needed addition of Ceftriaxone after five days (Table 2). The average fever defervescence time in Ceftriaxone group was 3.88 days and in Ofloxacin group was 3.04 days.All patients were afebrile by 9 days of antibiotics. Average duration of hospital stay for patients on Ceftriaxone group was 6.64 days and on Ofloxacin group was 5.28 days. There were no complications and all patients were discharged from the hospital in stable condition.

\section{DISCUSSION}

Enteric fever also known as enteric feveris a systemic illness characterized by high grade fever and abdominal symptoms and it makes a major portion of hospital admission diagnosis for febrile patients in our hospital. Though enteric feveris usually treated with a single antibacterial drug, the optimal choice of drug and duration of therapy is uncertain and the selection of antibiotics depend upon local resistance patterns, patient age, whether oral medications are feasible, the clinical setting, and available resources ${ }^{6-8}$. The current recommendation for the treatment is with either fluoroquinolone such as ciprofloxacin or oflaxacin or with third generationcephalosporins such as ceftraxone ${ }^{9}$. The emergence of multi drug resistance (MDR) strains have caused numerous outbreaks in the Indian subcontinent, Southeast Asia, Mexico, the Arabian Gulf, and Africa ${ }^{4,10}$. There are concerns with the resistance even with ofloxacin and ceftriaxone $e^{4-5}$. In this study, we tried to see the response of enteric fever in terms of fever defervescene with Ofloxacin, Cefriaxone or both.

The common presenting symptoms of enteric fever which are fever, abdominal pain, headache, cough, diarrhea and constipation were consistent with other previous studies. In a study conducted 


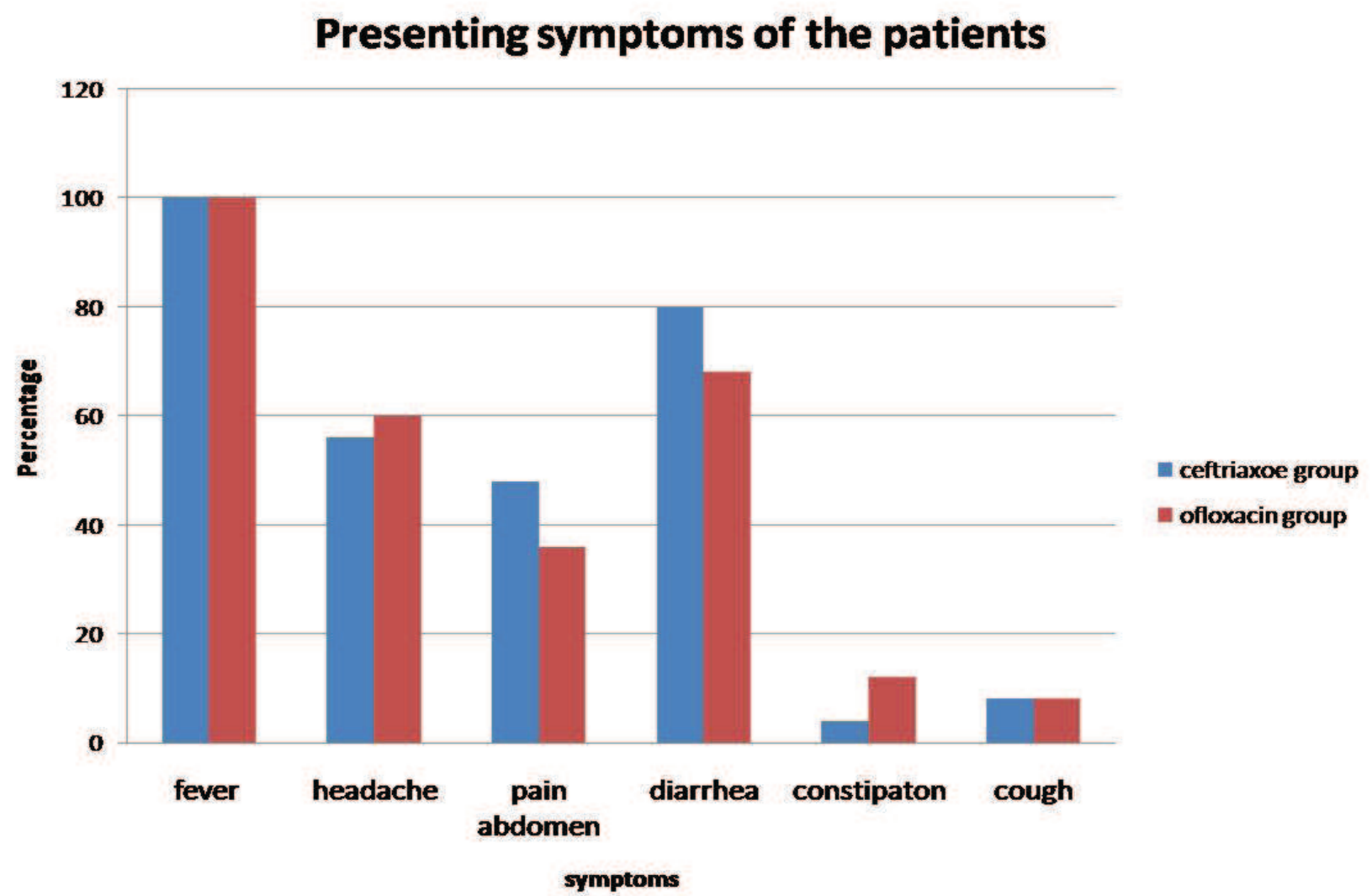

Table 1: Baseline characteristics of the two treatment groups

\begin{tabular}{|l|c|c|c|}
\hline Variables & $\begin{array}{c}\text { Ceftriaxone group } \\
(\mathrm{n}=25)\end{array}$ & $\begin{array}{c}\text { Ofloxacin group } \\
(\mathrm{n}=25)\end{array}$ & $\mathrm{p}$ value \\
\hline Mean age & 28.92 & 31.36 & 0.45 \\
\hline Sex (male) & $24(96 \%)$ & $21(84 \%)$ & 0.34 \\
\hline Fever duration at presentation (days) & 8.6 & 6.04 & 0.70 \\
\hline Headache & $14(56 \%)$ & $15(60 \%)$ & 1.00 \\
\hline Abdominal pain & $12(48 \%)$ & $9(36 \%)$ & 0.56 \\
\hline Diarrhoea & $20(80 \%)$ & $17(68 \%)$ & 0.52 \\
\hline Constipation & $1(4 \%)$ & $3(12 \%)$ & 0.62 \\
\hline Cough & $2(8 \%)$ & $2(8 \%)$ & 1.00 \\
\hline Blood culture positivity & $2(8 \%)$ & $3(12 \%)$ & 1.00 \\
\hline
\end{tabular}

by David et $\mathrm{al}^{11}$ in CIWEC clinic of Nepal and by Sharma et $\mathrm{al}^{12}$ in Dhulikhel have shown the similar clinical features of typhoid fever.

In view of poor result from the blood culture, which was only $5 \%$ of total cases, we had to depend mostly on the clinical features of typhoid fever. The low yield of culture may be due to inappropriate use of antibiotics by the primary care health worker before the patient presents to the hospital and the delay in incubating the media after the blood withdrawal. 
Table 2. cross tabulation between treatment group and addition of second drug

\begin{tabular}{|c|c|c|c|}
\hline \multirow{2}{*}{ Treatment Group } & \multicolumn{2}{|c|}{ Second drug added } & Total \\
\hline & No & Yes & 25 \\
\hline Ceftriaxone & 19 & 6 & 25 \\
\hline Ofloxacin & 23 & 2 & 50 \\
\hline Total & 42 & 8 & \\
\hline
\end{tabular}

The average fever defervescence time in Ceftriaxone group was 3.88 days and in Ofloxacin group was 3.04 days which was also consistent with the similar studies of enteric fever done in Kathmandu ${ }^{12}$. In our study, six of the patients receiving ceftriaxone and two of those receiving ofloxacin needed the addition of the second drug which was ofloxacin in ceftriaxone group and vice versa. The study done by Sharma et al ${ }^{12}$ in Dhulikhel hospital had shown $100 \%$ sensitivity to the ceftriaxone however the present finding of our study showed different result. Smith et al had shown in his study when comparing the treatment with oral ofloxacin with intravenous ceftriaxone in Vietnamese patients, that short-course treatment with oral ofloxacin was significantly better than that withceftriaxone ${ }^{13}$.

The low number of sample size and the lack of correlation whether the non responder were having resistant to the treatment drug in vitro also have been the limitation of our study. However it does provide a new area of research for the emerging resistance pattern of the salmonella species in our part of the world. It also raises the concern whether multi drug therapy may be better than monotherapy in order to avoid treatment failure or drug resistance.

\section{CONCLUSION}

Fever defervescence time with Ofloxacin was lesser than ceftriaxone group and need of supplementary antibiotic is lesser in ofloxacine group. So, ofloxacin can be recommended as $1^{\text {st }}$ line drug for enteric fever. Further large randomised trials are needed to substantiate the findings.

\section{REFERENCES}

1. Parry CM, Hien TT, Dougan G, et al. Typhoid fever. N Engl J Med 2002; 347:1770.

2. Osler W. The principles and practice of medicine: designed for the use of practitioners and students of medicine. 8th edn. New York:D Appleton, 1912: 1-46.

3. Crump JA, Luby SP, Mintz ED. The global burden of typhoid fever.Bull World Health Organ 2004; 82: 346-53.

4. Rowe B, Ward LR, Threlfall EJ. Multidrug-resistant Salmonella typhi: a worldwide epidemic. Clin Infect Dis 1997; 24 (suppl 1): S106-09.

5. Saha SK, Talukder SY, Islam M, Saha S. A highly ceftriaxone resistant Salmonella typhiin Bangladesh. Pediatr Infect Dis J 1999; 18: 387.

6. Wain, J, Hoa, NT, Chinh, NT. Quinolone-resistant Salmonella typhi in Viet Nam: Molecular basis of resistance and clinical response to treatment. Clin Infect Dis 1997; 25:1404.

7. Limson, BM. Short course quinolone therapy of enteric feverin developing countries. Drugs 1995; 49 S2:136.

8. Thaver, D, Zaidi, A, Critchley, J, et al. Fluoroquinolones for treating typhoid and paraenteric fever(enteric fever). Cochrane Database Syst Rev 2005; :CD004530.

9. Longo DL, Kasper DL, Jameson LJ, Fauci AS, Hauser LS, Loscalzo J. Harrison's Principles of Internal Medicine. 18th ed. New York:McGrawHill; 2012:1274-81.

10. RastegarLari A, Validi N, Ghaffarzadeh K, Shamshiri AR. In vitro activity of cefixime versus ceftizoxime against Salmonella typhi. PatholBiol (Paris) 1997; 45:415.

11. David R. Shlim,Eli Schwartz,Molly Eaton.Clinical Importance of Salmonella Paratyphi A Infection to Enteric Fever in Nepal.Journal of Travel Medicine.1995;2(3):165-68

12. Sharma N, Koju R, Karmacharya B, Tamang MD, Makaju R, Nepali N, Shrestha P, Adhikari D.Typhoid fever in Dhulikhel hospital, Nepal.Kathmandu Univ Med J (KUMJ). 2004 Jul-Sep;2(3):188-92.

13. Smith MD,DuongNM,HoaNTT,WainJ,HaHD,Diep TS et al. Comparison of Ofloxacin and Ceftriaxone for ShortCourseTreatment of Enteric Fever. Antimiocrobs agents chemother. 1994; 38(8):1716-20 